\title{
ERRATUM
}

\section{Microbiology turns inwards}

[No authors listed]

Nature Reviews Microbiology 9, 224 (2011)

In this Editorial, we mistakenly credited the Human Genome Project instead of the Human Microbiome Project in the efforts to understand human microbiome. The text should have read: "The coordinated efforts of the Human Microbiome Project (sponsored by the US National Institutes of Health) and the MetaHIT project (sponsored by the Seventh Framework Programme of the European Commission) have taken advantage of the latest sequence technologies to reveal in great detail the composition of the human gut microbiota, and similar projects are focusing on other body sites."

We apologize to researchers involved in the Human Microbiome Project and to the readers for any confusion caused. 